\title{
MORPHOLOGY OF THE JUPITER IO-D DECAMETRIC RADIO SOURCE
}

\author{
C. Higgins*, T. E. Clarke†, K. Imai ${ }^{\ddagger}$, M. Imai ${ }^{\S}$, F. Reyes $\mathbb{I}$, and J. Thieman
}

\begin{abstract}
Observations of Jupiter's Io-D decametric radio emissions have been made with the Long Wavelength Array Station 1 (LWA1) from 2012-2015. The LWA1 data show new characteristics in dynamic spectra of Jupiter Io-D emission, including a double-envelope structure filled with spectral arc features, persistent narrowband events, and ubiquitous modulation lanes and S-bursts. We observe an Io-D peak frequency of $26.5 \mathrm{MHz}$ implying a lower limit of 9.3 Gauss for the southern hemisphere magnetic field magnitude. We estimate a Jovian longitude of $235^{\circ}$ for the center location of the Io-D peak frequency source. Many S-burst drift rates were measured as a function of frequency and were found to be consistent with other Iorelated sources. The Io-D source occurrence boundaries require new limits: CML $=0^{\circ}-287^{\circ}$ and $\Phi_{I o}=50^{\circ}-135^{\circ}$.
\end{abstract}

\section{Introduction}

Jupiter radio emission has been observed by ground-based observatories since the discovery in 1955 by Burke and Franklin [1955]. The decameter radio emission (DAM) ranges from about $3 \mathrm{MHz}$ to $39.5 \mathrm{MHz}$, and these emissions have been used to characterize and define Jupiter's magnetic field, the internal System III rotation period, the general emission mechanism, polarization, spectral features, and the approximate source locations (for reviews, see Carr et al. [1983]; Zarka, [1998]; Clarke et al., [2004]). It is also well-known that the satellite Io influences much of the DAM emission [Bigg, 1964], and some nonIo-related emissions are correlated with the solar wind [Barrow et al., 1986; Hess et al., 2012].

\footnotetext{
* Department of Physics and Astronomy, Middle Tennessee State University, Murfreesboro, TN, USA

${ }^{\dagger}$ Remote Sensing Division, US Naval Research Laboratory, Washington, DC, USA

$\ddagger$ Department of Elect. Engineering and Information Sci., Kochi National College of Technology, Kochi, Japan

$\S$ Department of Physics and Astronomy, University of Iowa, Iowa City, IA, USA

$\mathbb{I}$ Department. of Astronomy, University of Florida, Gainesville, Florida, USA

"University of Maryland Baltimore County, Baltimore, MD, USA
} 
The Io-A, Io-B, and Io-C sources were initially labeled A, B, and C, or "main", "early", and "late", respectively [Carr et al., 1961; Dulk, 1965]. Warwick [1963] predicted a fourth source and Dulk [1965] first identified it; soon thereafter Gledhill [1967] used the term "Fourth Source" to identify the Io-D component and specified the observed range of Io-D as CML $0^{\circ}-70^{\circ}$ and Io-Phase $\left(\Phi_{\text {Io }}\right) 90^{\circ}-110^{\circ}$. Kennedy [1969] determined the LH polarization sense of the Io-D source. Figure 1 shows a general overview of the four Io-related sources and their approximate location in Jupiter's magnetosphere.

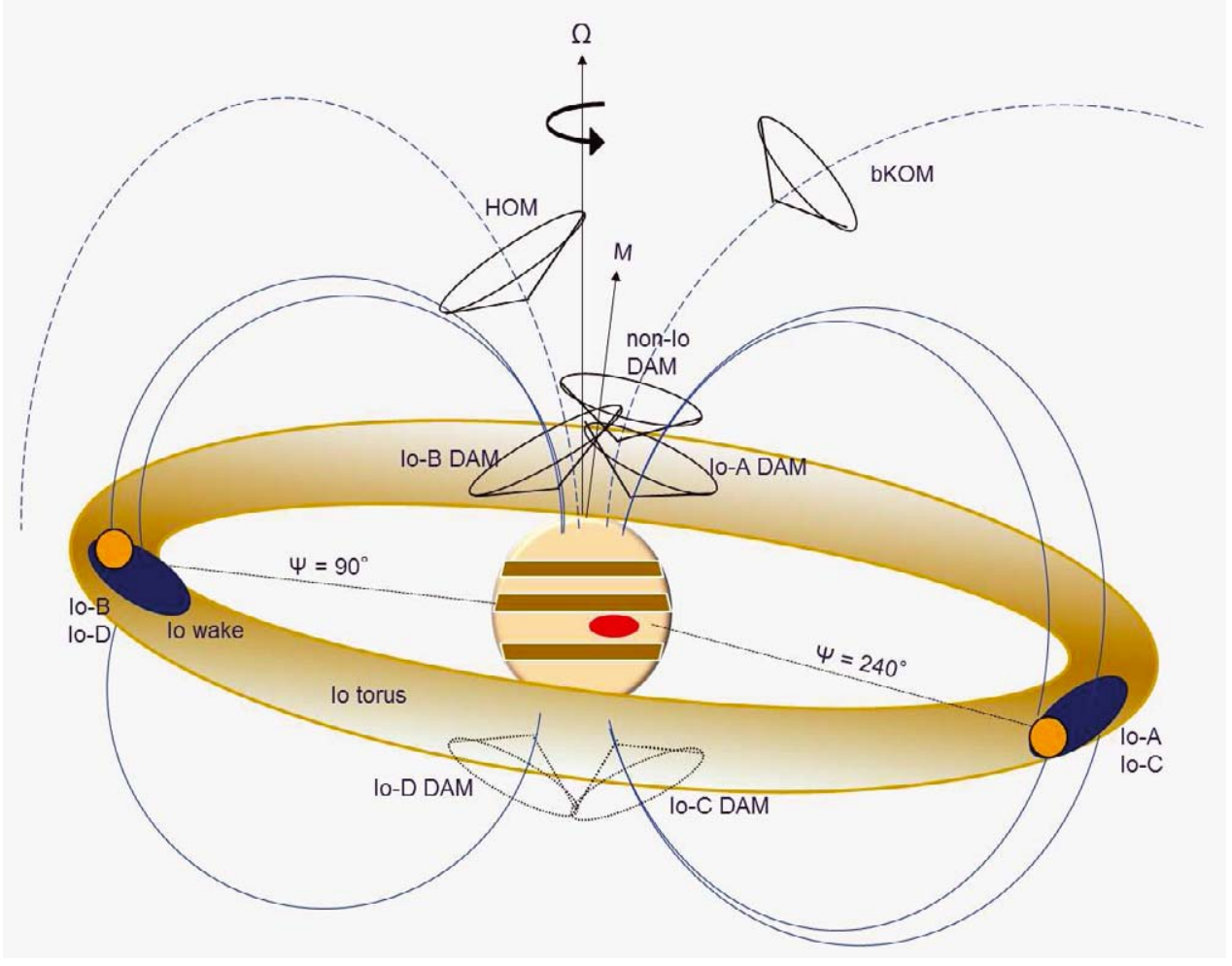

Figure 1: Most of Jupiter's auroral radio emission sources are labeled here: bKOM - broadband kilometric emission, HOM - hectometric emission, non-Io-DAM - decametric emission related to HOM not controlled by Io, and Io-DAM - decametric emission tied to Io flux tube and Io torus. Also seen are the approximate phases of Io $\left(\Phi_{I o}\right)$ for the Io-related sources (adapted from J. Clarke et al. [2004]).

From the summary of Carr et al. [1983], the Io-D source was known to be left-hand circularly polarized (LHC), show vertex early arc structure having a maximum frequency of $18 \mathrm{MHz}$, and to occur within the CML range $0^{\circ}-200^{\circ}$ and $\Phi_{\text {Io }} 95^{\circ}-130^{\circ}$. DAM emission is believed to be generated by the cyclotron maser instability (CMI) mechanism and dominated by X-mode emission emitted near the local cyclotron frequency; therefore, because it is LHC polarized the location of the Io-D source is presumably in Jupiter's southern hemisphere. Because the Io phase-CML range of Io- $\mathrm{D}$ is similar to the Io-B source, it is considered to be the complement to the northern hemisphere Io-B source. Not many spectral details were known about the Io-D source before the Voyager flybys because the Io-B emission occurs at the same times and is much more dominant. Leblanc [1981] used Voyager observations of Jupiter DAM and found that Io-D had a narrower band of emission, as compared with the other Io-related sources, and appeared as a long 
envelope of a vertex-early arc with possible arc-like substructure. She also observed Io$\mathrm{D}$ from $5-22 \mathrm{MHz}$ and found that Io-D had a CML range $0^{\circ}-260^{\circ}$ and a $\Phi_{\text {Io }}$ range $95^{\circ}-140^{\circ}$.

Genova and Aubier [1985] found the high frequency limits of the Io-related emissions using Voyager data. They compared those limits to the surface gyrofrequency of a magnetic model and deduced that the Io-D emission comes from the field line passing through Io. They show evidence of Io-D emission at nearly all longitudes. Wilkinson [1989] also used Voyager data and an empirical technique to model some of the Io-related spectral structures, including the Io-D source requiring an $86^{\circ}$ beaming cone half angle. Leblanc et al. [1993] used the Voyager 1 and 2 flyby data to study Jupiter's LH polarized DAM emission. They found that the occurrence probabilities of LH (and RH) emission peak at about $8 \mathrm{MHz}$, but that the LH probability diminishes significantly above $15 \mathrm{MHz}$. They found that the probability spectra in CML show a continuity from low to high frequencies, and that the emission extends down to $2.5 \mathrm{MHz}$. They argued that the emission was highly beamed in hollow cones with large opening angles, and that Io influenced the emission at all longitudes for $\nu<10 \mathrm{MHz}$.

Boudjada and Genova [1991] studied LH decameter events in Nançay spectrograph data and concluded that the maximum frequency for LH events (both Io and non-Io) is $25 \mathrm{MHz}$ which sets a limit of about 9 Gauss for the maximum field strength of Jupiter's southern hemisphere. Dulk et al. [1994] analyzed the polarization state of Io-related storms and found that the emission was $100 \%$ elliptically polarized showing little variation over a long period of time. They found that the degree of circular polarization was very high (0.85) for Io-D events. Leblanc et al. [1994] studied Io-D and Io-B LH emission events and identified them as distinct LH southern hemisphere emission sources. Queinnec and Zarka [1998] used combined Nanąy and Wind spacecraft data and found Io-D as isolated arcs from approximately 3-23 $\mathrm{MHz}$ in frequency-time spectrograms, and show that Io-D shows more complex structure at the higher frequencies. Like other Io-related sources, they find the arc structure is strongly dependent on viewing geometry. Their data and model show that Io-D is a southern hemisphere source having LHC polarization with a beaming cone half-angle of $70^{\circ}-85^{\circ}$ and an envelope shape that is caused by a source along southern Io-flux tube in Io's frame. Their Io-D events fall in the range of CML $55^{\circ}-200^{\circ}$ and $\Phi_{I o} 80^{\circ}-110^{\circ}$.

As mentioned before, many fine spectral details of the Io-D source were not known because of the overlap with the more powerful Io-B source. This is also true for the millisecond "short" S-burst structures, and before the Clarke et al. [2014] paper only one published Io-D S-burst event was known [Litvinenko et al., 2010]. However, it is likely that Dulk et al. [1994] observed S-bursts related to the Io-D source but attributed it to the Io-B source. No statistics specific to the Io-D source are known. Dulk et al. [1994] measured the complete polarization state of both left-hand and right-hand S-bursts from various sources and found that the LH S-bursts were highly circularly polarized, having a degree of circular polarization $d_{c}=$ Stokes $\mathrm{V} / \mathrm{I}=+0.85$ (the $\mathrm{RH}$ events had $d_{c}=+0.55$ ). Queinnec and Zarka [2001] did a statistical analysis of mostly RH S-bursts and found an emission bandwidth of 0.5-9.0 MHz, a 1 AU normalized average flux density of $4 \times 10^{-20} \mathrm{~W} \mathrm{~m}^{-2} \mathrm{~Hz}^{-1}$, and an average radiated power of $\sim 10^{9} \mathrm{~W}$. Hess et al. [2007] measured frequency drifts 
of S-bursts and determined that a typical energy of the emitting electrons is $4 \mathrm{keV}$, and also showed that the drift rates decrease at high frequencies in accordance with adiabatic theory. Recently, Imai et al. [2016] used simultaneous observations of S-bursts from three radio telescopes to constrain the beaming thickness to 2.66 ". They also found that the occurrence probability of S-bursts peaks at 17-18 MHz. Much more information about Jovian S-bursts can be found in the literature (i.e., Arkhypov and Rucker [2009]; Ryabov, et al. [2007]; Zarka et al., [1996]; Flagg et al. [1991]; Carr et al. [1983]).

\section{Observations and data analysis}

Observations of Jupiter were conducted from 2012 to 2015 using the Long Wavelength Array Station 1 (LWA1) located in Magdalena, NM, USA, near the site of the VLA. The array consists of 256 inverted $\mathrm{V}$-shaped crossed-bowtie dipoles having all-sky viewing capability from 10-88 MHz. The system operates with 4 independent beams having a beam size of $8.1^{\circ}$ at $20 \mathrm{MHz}$, full Stokes parameters, and a usable bandwidth of $16 \mathrm{MHz}$. We observed Jupiter with two tunings over the frequency range of $10-38 \mathrm{MHz}$ with a resolution of $5 \mathrm{kHz}$ and $0.21 \mathrm{~ms}$. To ensure accurate polarization determinations, observations were limited to Jupiter elevations above $40^{\circ}$. For more information about the LWA1 instrument see Clarke et al. [2017] and Clarke et al. [2014] and references therein. The LWA1 instrument is an excellent instrument for low frequency radio studies, and the spectrum is reasonably clean down to $10.5 \mathrm{MHz}$ as can be seen in Figure 2 showing excellent Io-B and Io-D Jupiter emission. The right-hand circular (RHC) Io-B emission is shown in red color while the LHC emission is shown in blue. Fine spectral structure is clearly seen in both sources.

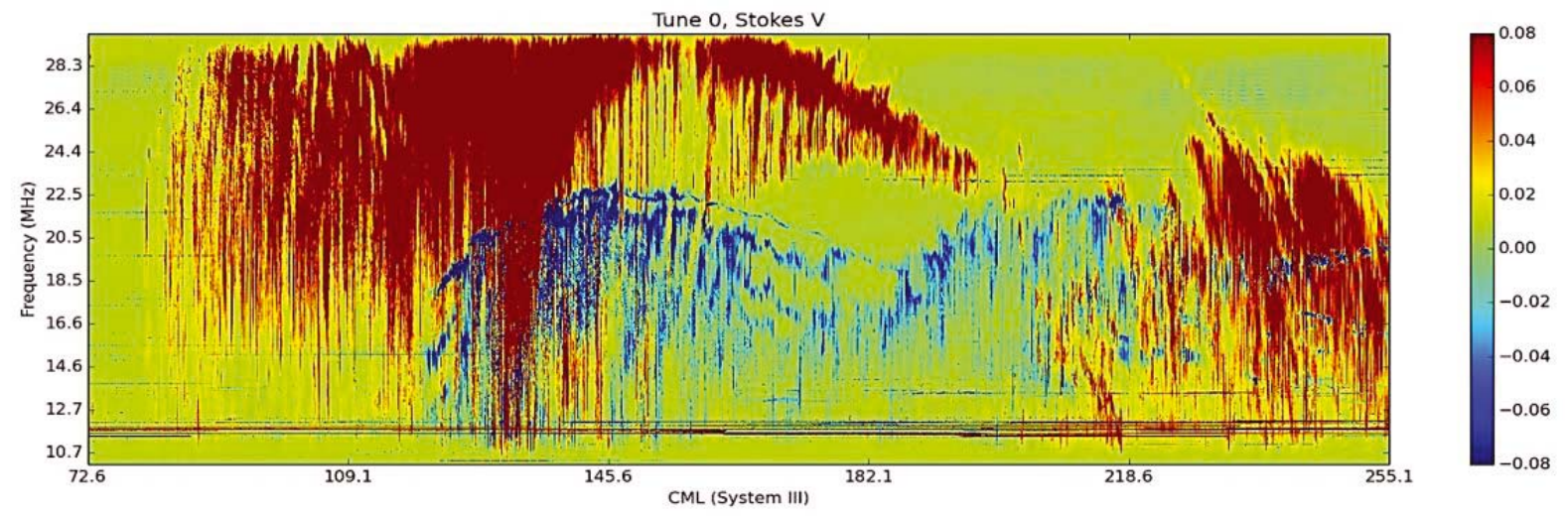

Figure 2: Jupiter Io-B/Io-D emission on 20 January, 2015 beginning at 05:30 UT. Io-B followed by non-Io-A emission is right-hand circularly polarized (red) and Io-D is left-hand circularly polarized (blue). The 5-hour observation shows a frequency range from 10.35 to $29.65 \mathrm{MHz}$, a $C M L$ range of $72.6^{\circ}-255.1^{\circ}$, and a corresponding $\Phi_{I o}$ from $80^{\circ}-123^{\circ}$. The colorbar shows Stokes $V$ power spectral density in arbitrary units.

Although Jupiter observations with the LWA1 were conducted from 2012-2015, the Io-D events shown here are selected from 2014-2015. These data are recorded as raw voltages and then processed through a Fourier transform spectrometer using LWA software tools 
[Dowell et al., 2012]. Data are converted from linear polarization to R, L and Stokes parameters. A typical observation was $3-5$ hours in duration and initially processed at $2 \mathrm{~s}$ and $10 \mathrm{kHz}$ resolution (Figures 2, 3, 4). Selected 5-second subsets of these data were processed at $0.25 \mathrm{~ms}$ and $5 \mathrm{kHz}$ resolution to analyze fine structures. Occasionally a lower resolution spectrometer mode (40 ms and $20 \mathrm{kHz}$ ) was used to observe Jupiter emissions. For more details about the LWA1 Jupiter data analysis procedure, see Clarke et al. [2014].

\section{Characteristics of the Io-D radio source}

As mentioned in the Introduction, the Io-D radio source has been known since the mid1960s. Improved receivers, antennas, and software have made it much easier to observe polarization signatures and fine structures in Jovian dynamic spectra. The Io-D decametric radio source is quite easy to detect in spectra of Stokes $\mathrm{V}$ as seen in Figures 2, 3, and 4. Io-D shows a typical envelope of emission, comprised of vertex-early arc structures that increase in frequency from $<10 \mathrm{MHz}$ to a peak of $20-24 \mathrm{MHz}$ at about $140^{\circ}-150^{\circ} \mathrm{CML}$ and then decrease in frequency until the emission ends. From twelve Io-D events from 2012-2015 where more than $50 \%$ of the envelope is seen, we determined that the average peak frequency of the Io- $\mathrm{D}$ source is $22.8 \mathrm{MHz}$, and the average observed position of the peak frequency is $154^{\circ} \mathrm{CML}$ and $99^{\circ}$ Io phase. Queinnec and Zarka [1998] determined a beaming angle for Io-D of $80^{\circ} \pm 5^{\circ}$ from arc structures and a field model. Using a symmetric hollow cone beam shape and Jovian ephemeris information, the calculated averaged position of the maximum frequency of Io-D source is approximately $235^{\circ}$ in Jovian longitude. This value represents the approximate Jovian longitude position of the Io-D emission source, in good agreement with the study of Genova and Aubier [1985].

The envelope and arc structure are defined by the beaming geometry of the southern hemisphere source [Leblanc et al., 1993; Leblanc, 1981]. However, as seen in Figures 2 and 3, a second envelope of emission appears before the first has dissipated reaching nearly the same maximum frequency. This argues for an extended source region in the southern hemisphere. Hess et al. [2008] were able to simulate many of the Io-related arc structures using an emission beam model, the lead angle between Io and the emitting field line, and the electron energy. In particular, with a very small lead angle, $+8^{\circ}$, they were able to show observed arc structures for the Io-D source. However, there were discrepancies in the peak frequencies simulated, most likely due to inaccuracies in the Jovian field model near the planet. Previous ground-based and Voyager data show that Io-D emission extends from $0^{\circ}-260^{\circ}$ [Leblanc, 1981], and Figure 2 shows that the Io-D emission may extend to higher longitudes. The Io-D emission splits into three or four distinct bands of emission, and in some cases the bandwidth $<100 \mathrm{kHz}$. These narrow-band features are N-events having typical bandwidths of $200-600 \mathrm{kHz}$ over many tens of minutes. Figure 4 shows an Io-D envelope that narrows into an N-event and persists for more than 1 hour. Many of these spectral structures are similar to those seen in the Io-B source, and perhaps require similar explanations for the beaming [e.g. Queinnec and Zarka, 1998].

Figure 5 shows an Io-D event that is interesting for two reasons. First the maximum frequency of emission is $26.5 \mathrm{MHz}$, higher than previously known. Marques et al. [2017] have presented some evidence that Io-D emission reach $27.9 \mathrm{MHz}$ from observations with the 


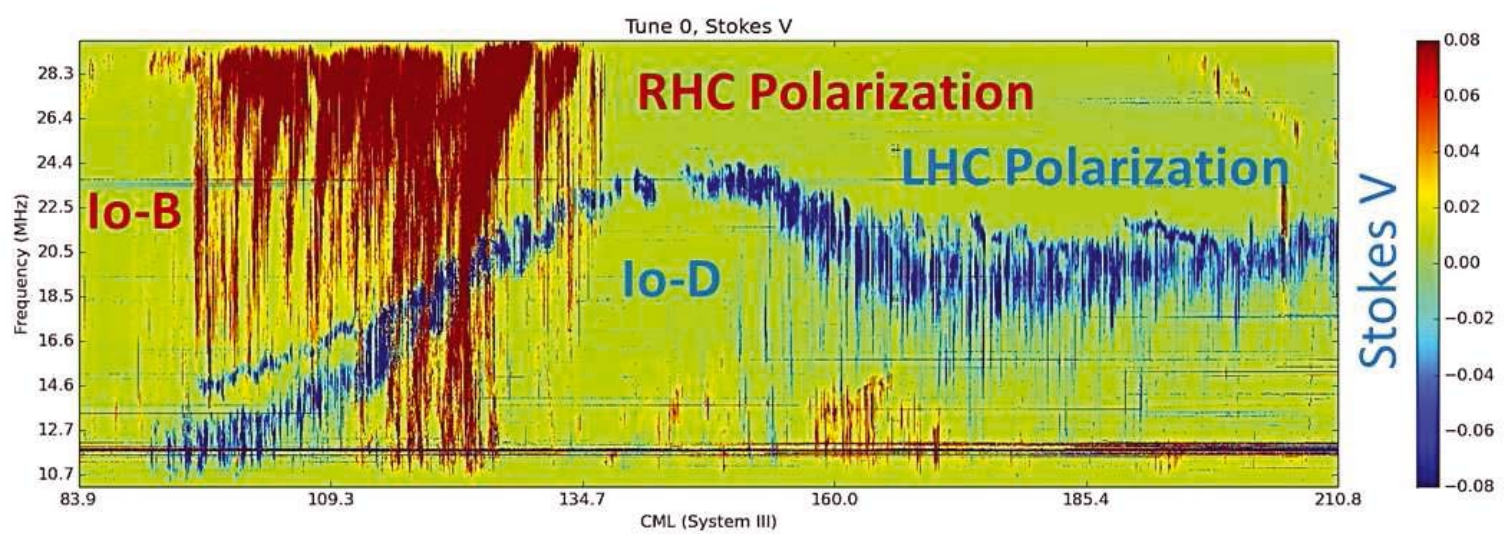

Figure 3: Jupiter Io-B/Io-D emission on 12 December, 2014 beginning at 09:00 UT. The 3.5-hour observation shows a frequency range from 10.35 to $29.65 \mathrm{MHz}$, a $C M L$ range of $83.9^{\circ}-210.8^{\circ}$, and a corresponding $\Phi_{I o}$ from $90^{\circ}-120^{\circ}$. The colorbar shows Stokes $V$ power spectral density in arbitrary units.

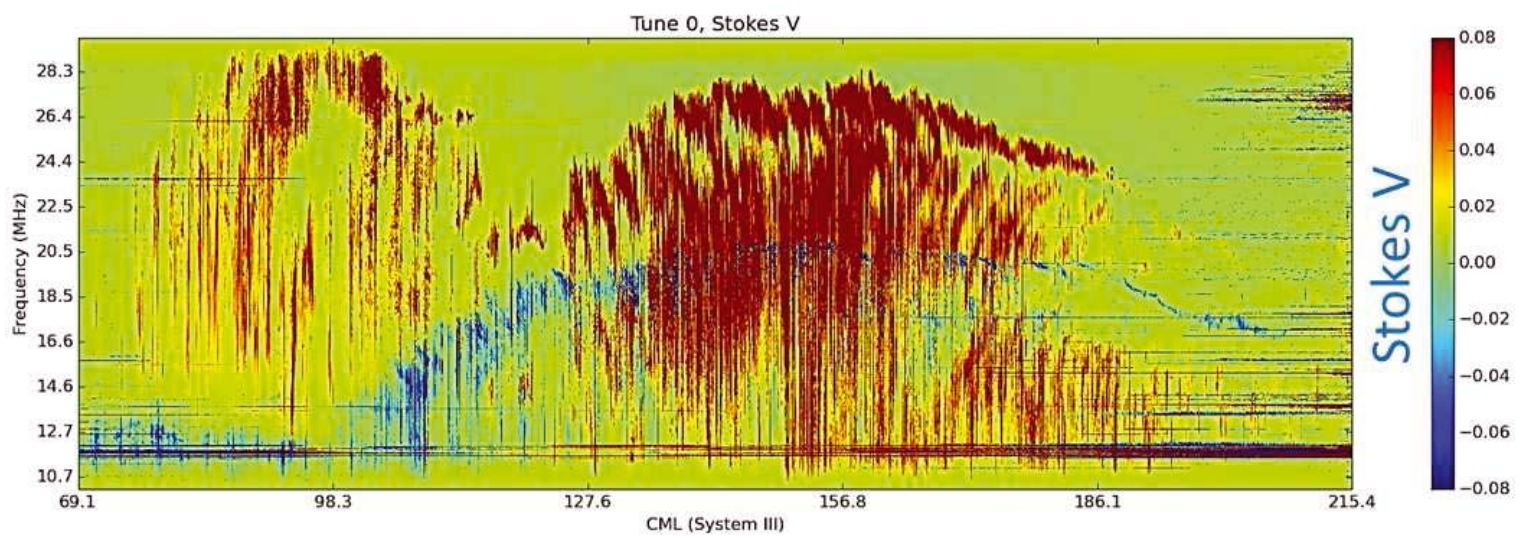

Figure 4: Jupiter Io-B/Io-D emission on 26 December, 2014 beginning at 10:00 UT. The 4-hour observation shows a frequency range from 10.35 to $29.65 \mathrm{MHz}$, a CML range of $69.1^{\circ}-215.4^{\circ}$, and a corresponding $\Phi_{I o}$ from $68^{\circ}-102^{\circ}$. The colorbar shows Stokes $V$ power spectral density in arbitrary units.

Nana̧y Decameter Array. In either case, this extension to higher frequencies of a southern hemisphere radio source has profound implications on the maximum field strength in the southern hemisphere; for example, $\nu=26.5 \mathrm{MHz}$ requires a field strength of approximately 9.5 Gauss.

These LWA1 observations show Io-D in the CML range $67^{\circ}-262^{\circ}$ and in the $\Phi_{\text {Io }}$ range $68^{\circ}-135^{\circ}$. Typinski [private communication, 2015] has recorded Io-D up to CML $287^{\circ}$ and as low as $\Phi_{I o} 50^{\circ}$. Clearly, the radio source boundaries on the Io phase-CML occurrence probability map are frequency dependent. Therefore, using these data with the Leblanc (1981) Voyager results, the Io-D region observed from $2.5-26.5 \mathrm{MHz}$ on the Io phaseCML occurrence probability map should have the following limits: $\mathrm{CML}=0^{\circ}-287^{\circ}$ and $\Phi_{I o}=50^{\circ}-135^{\circ}$. In light of these new data, the limits of the Io-D region need 


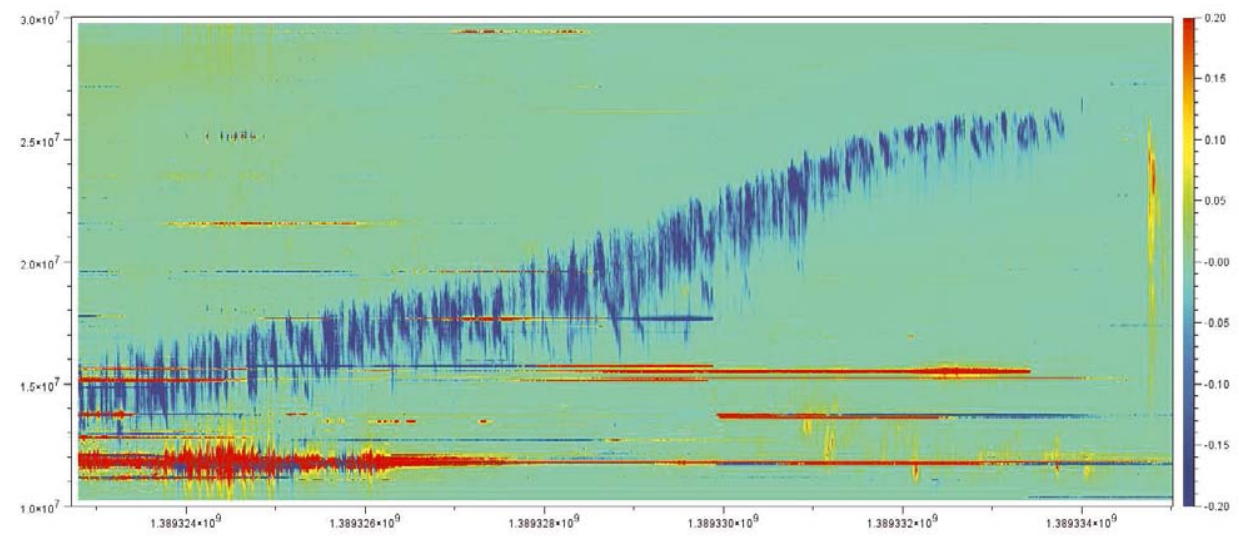

Figure 5: Io-D event on 10 January, 2014 from 03:00-06:24 UT $\left(C M L=86.7^{\circ}-210.1^{\circ}, \Phi_{I o}=\right.$ $106^{\circ}-135^{\circ}$ ) observed using LWA1 spectrometer mode at $40 \mathrm{~ms}$ and $20 \mathrm{kHz}$ resolution. The peak frequency is $26.5 \mathrm{MHz}$. The colorbar shows Stokes $V$ power spectral density in arbitrary units. Horizontal lines are interference with some saturation at $12 \mathrm{MHz}$.

redefinition.

Modulation lanes are very common features in Jupiter's Io-related emissions [Riihimaa, 1968; Imai et al., 2002]. Although not shown here, modulation lane structures are easily seen in the LWA1 observations at modest resolutions, $40 \mathrm{~ms}$ and $20 \mathrm{kHz}$ [Clarke et al., 2014]. From the current analysis of LWA1 data, modulation lanes are ubiquitous in the Io-D emission, as well as in the other Io-related source emission. These features can be used to place bounds on the source positions and beaming pattern of the radio emission [K. Imai et al., 2017; Imai et al., 2002]. The Io-D modulation lane events could lead to a better understanding of the southern hemisphere radio sources.

Previous detections of S-burst events in the Io-D source are few [Clarke et al., 2014; Litvinenko et al., 2010]. However, these data from LWA1 show that S-bursts are ubiquitous in the Io-D source. A subset of the LWA1 observations were analyzed for S-burst activity, and Figure 6 shows several examples of LH S-bursts within the Io-D source. In many cases, both RH S-bursts from the Io-B source are seen simultaneously. Although Io-D S-bursts are seen at all longitudes of the source region observed with the LWA1 $\left(67^{\circ}-262^{\circ}\right.$ $\mathrm{CML}$ ), their occurrence is higher between $67^{\circ}-150^{\circ} \mathrm{CML}$. That is, more S-bursts were found at the leading edge of the source indicating a strong initial interaction with Io as the source region sweeps past Io. This is consistent with the statistics of S-bursts from the Io-B source [Flagg et al., 1991]. However, this Io-D study is based on a small sample size and more data are needed to quantify this result.

The frequency-time drift rates of Jovian S-bursts have been used to determine the energies of the precipitating electrons, and, therefore, to test radio emission theories [e.g. Zarka et al., 1996]. Figures 6-9 show many S-bursts with a diversity of structure and differing drift rates. Figure 6 shows left-handed Io-D S-bursts between 14-18 MHz (blue) with complex structure, similar to known Io-B S-bursts, and also shows Io-B S-bursts (red) at higher frequencies. Frequency drift rates were measured for at least five S-bursts in each observation. The Io-D drift rates are $-12.8 \mathrm{MHz} / \mathrm{s}$ at $16 \mathrm{MHz}$, whereas the Io-B drift 


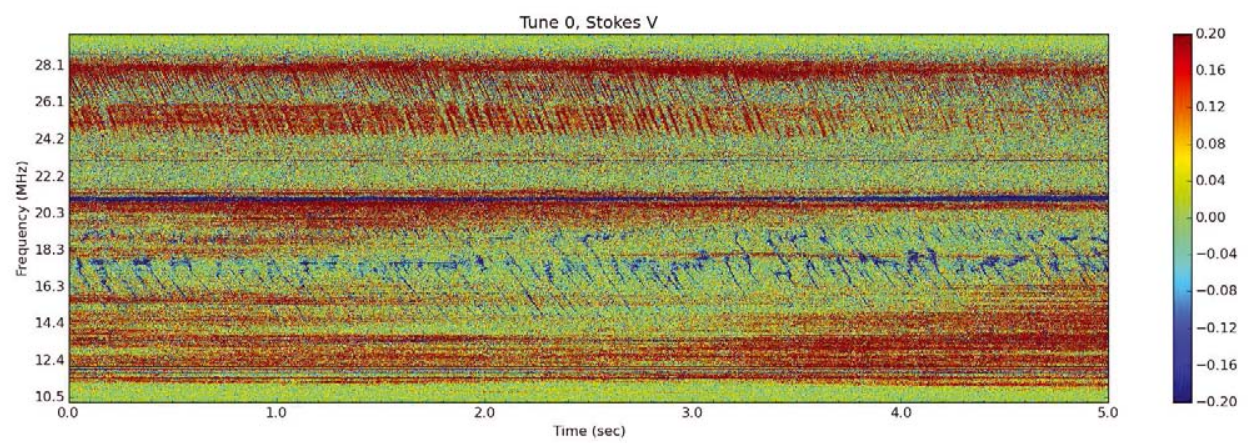

Figure 6: Io-D S-bursts (blue) and Io-B S-bursts (red) on 27 January, 2015 at 09:01 UT (CML $\left.=174.9^{\circ}, \Phi_{I o}=95.2^{\circ}\right)$. Note the different $S$-burst drift rates for the Io-B $(-21.1 \mathrm{MHz} / \mathrm{s})$ and the Io-D (-12.8 MHz/s) events.

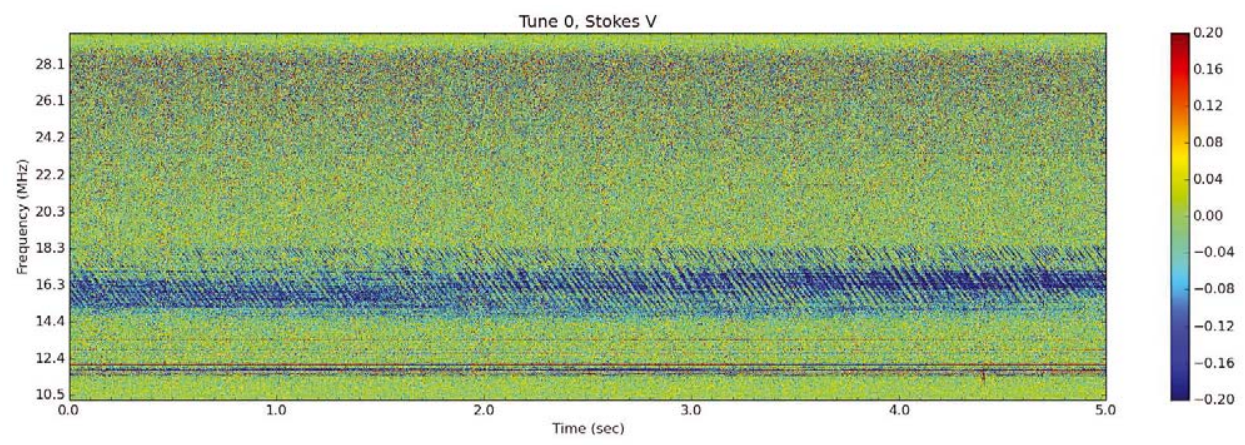

Figure 7: Io-D S-bursts on 27 January, 2015 at 10:19 UT $\left(C M L=222.2^{\circ}, \Phi_{\text {Io }}=106.3^{\circ}\right)$. The S-burst drift rates are $-13.8 \mathrm{MHz} / \mathrm{s}$ at $17.5 \mathrm{MHz}$.

rates are $-21.1 \mathrm{MHz} / \mathrm{s}$ at $26.5 \mathrm{MHz}$. The different drift rates of the Io- $\mathrm{B}$ and the Io-D sources demonstrate that these emissions are not co-spatial. Figure 7 shows a regular "train" of S-bursts in a clear band of emission between 15-18 MHz; the S-burst drift rates are $-13.8 \mathrm{MHz} / \mathrm{s}$ at $17.5 \mathrm{MHz}$. Figure 8 shows weak S-bursts at very low frequencies, down to $10.5 \mathrm{MHz}$; drift rates are $-8.2 \mathrm{MHz} / \mathrm{s}$ at $11.5 \mathrm{MHz}$. Finally, Figure 9 shows simultaneous S-bursts from both the Io-D and Io-B sources. The Io-D S-bursts are seen between $16.5-18.5 \mathrm{MHz}$ and have measured drift rates of $-9.2 \mathrm{MHz} / \mathrm{s}$ at $17.5 \mathrm{MHz}$. The drift rate data presented here, when plotted as a function of frequency, are consistent with the adiabatic scenario for generation (see Figure 3 from Zarka et al. [1996]). Again, the differing drift rates and polarization for the Io-D and Io-B S-bursts imply that the bursts are emitted from separate southern and northern hemisphere sources, respectively. An interesting question is whether the Io-B and Io-D S-burst events are related by common Alfvén waves or mirrored groups of electrons; this is left for future work.

\section{Conclusions}

The Long Wavelength Array (LWA1) is well-suited for studies of Jupiter decameter radio emission (DAM). It has a large bandwidth, full Stokes capability, and excellent resolution 


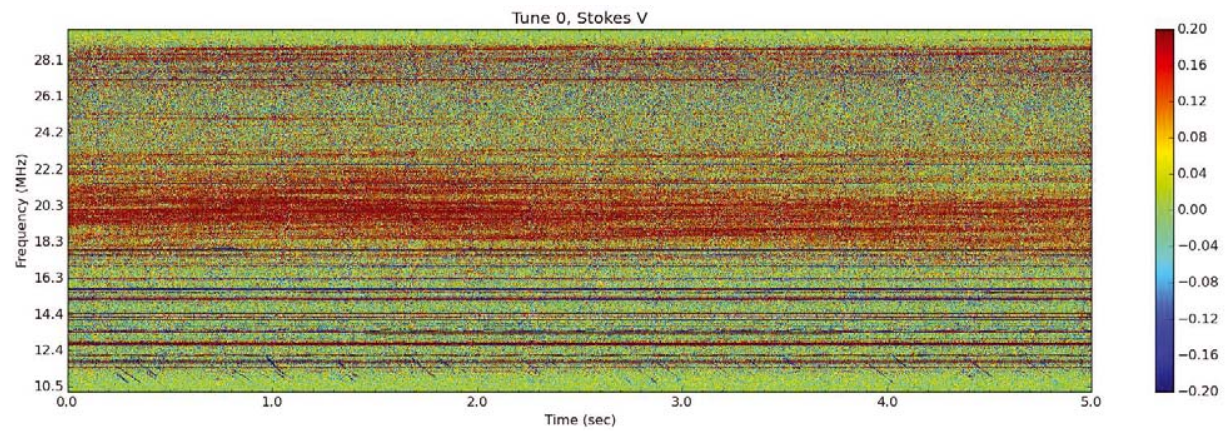

Figure 8: Io-D S-bursts on 28 February, 2015 at 02:49 UT $\left(C M L=91.0^{\circ}, \Phi_{I o}=78.3^{\circ}\right)$. The S-burst drift rates are $-8.2 \mathrm{MHz} / \mathrm{s}$ at $11.5 \mathrm{MHz}$.

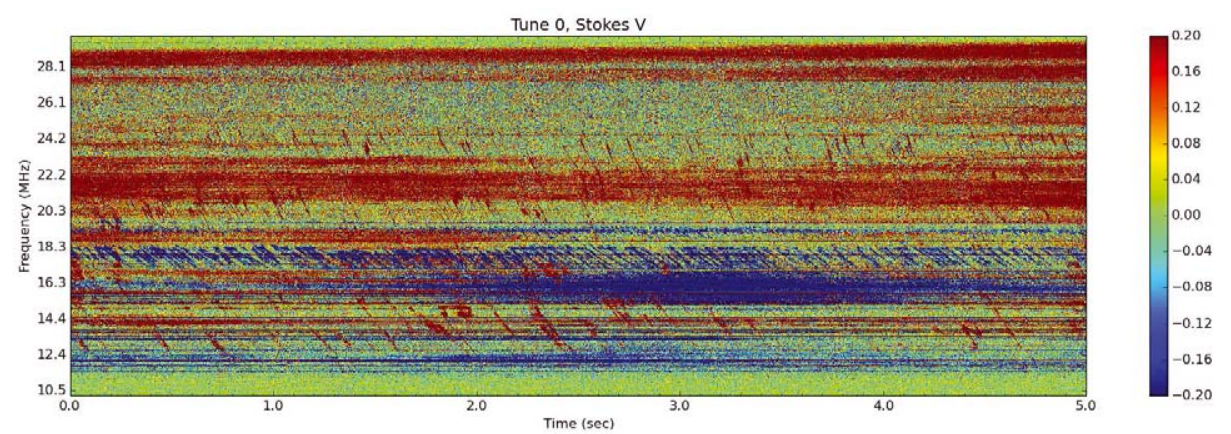

Figure 9: Io-D S-bursts on 07 March, 2015 at 04:43 UT $\left(C M L=134.2^{\circ}, \Phi_{I o}=79.5^{\circ}\right)$. The Io-D S-burst drift rates are $-9.2 \mathrm{MHz} / \mathrm{s}$ at $17.5 \mathrm{MHz}$. Io-B bursts occur from $12-15 \mathrm{MHz}$ (drift rate of $-11.6 \mathrm{MHz} / \mathrm{s}$ ) and $21-24 \mathrm{MHz}$ (drift rate of $-21.0 \mathrm{MHz} / \mathrm{s})$.

to study Jupiter's fine DAM structures. The Io-D radio source is clearly seen in LWA1 observations and shows characteristic LH polarization, and a sweeping envelope of vertexearly arc structures with substructures. We have presented new morphology attributed to the Io-D emission source. Our results about the Io-D source can be summarized as follows:

- We observe splitting of the main arc envelope into narrow banded events, some persisting for more than one hour.

- We observe a double-envelope structure, implying an extended southern hemisphere source region.

- The peak frequency of Io-D is $26.5 \mathrm{MHz}$; this requires a lower limit of approximately 9.3 Gauss for the magnitude of the southern magnetic field.

- The average peak frequency source location is estimated to be $235^{\circ}$ in Jovian longitude; this result can be used to further test magnetic field models.

- Modulation lanes are commonly seen in Io-D data. 
- S-bursts are ubiquitous in the Io-D source, and drift rates are consistent with an adiabatic emission scenario.

- More S-bursts are observed on the leading edge of the Io-D source implying a stronger interaction with Io at that time.

- The Io-D source occurrence boundaries require new, expanded limits: CML $=0^{\circ}-$ $287^{\circ}$ or greater (see Genova and Aubier [1985]) and $\Phi_{I o}=50^{\circ}-135^{\circ}$.

Acknowledgments: C.A.H. acknowledges support from the Tennessee Space Grant Consortium and from the Theodore Dunham, Jr. Grant of the Fund for Astrophysical Research. We also wish to thank the staff of the Long Wavelength Array. Basic research in Radio Astronomy at the US Naval Research Laboratory is supported by 6.1 Base Funding. This research has been supported in part by JSPS KAKENHI grant 16K05570. Construction of the Long Wavelength Array Station 1 (LWA1) was supported by the Office of Naval Research under contract N00014-07-C-0147. Support for operations and continuing development of LWA1 is provided by the National Science Foundation under grants AST-1139963 and AST-1139974 of the University Radio Observatories program. The Editors thank Sébastien Hess and one anonymous reviewer for their help in evaluating this paper.

\section{References}

Arkhypov, O.V., and H. O. Rucker, Regularity in dynamic spectra of Jovian radio decameter S-bursts, Astron. Astrophys., 496, 269-274, 2009.

Barrow, C.H., F. Genova, and M.D. Desch, Solar wind control of Jupiter's decametric radio emission, Astron. Astrophys., 165, 244-250, 1986.

Bigg, E. K., Influence of the satellite Io on Jupiter's decametric emission, Nature, 203, 1008-1010, 1964.

Boudjada, M. Y., and F. Genova, The left-hand polarization sense of the Jovian decameter radiation, Astron. Astrophys. Suppl. Ser., 91, 2, 453-467, 1991.

Burke, B. F., and K. L. Franklin, Observations of a variable radio source associated with planet Jupiter, J. Geophys. Res., 60, 213-217, 1955.

Carr, T. D., A. G. Smith, H. Bollhagen, N. F. Six, and N. E. Chatterton, Recent decameterwavelength observations of Jupiter, Saturn and Venus, Astrophys. J., 134, 105-125, 1961.

Carr, T.D., M. D. Desch, and J. K. Alexander, Phenomenology of magnetospheric radio emissions, in Physics of the Jovian Magnetosphere, edited by A. J. Dessler, Cambridge University Press, New York, USA, 226-284, 1983.

Clarke, J. T., D. Grodent, S. W. H. Cowley, E. J. Bunce, P. Zarka, J. E. P. Connerney, and T. Satoh, Jupiter's aurora, in Jupiter: the Planet, Satellites, and Magnetosphere, 
edited by F. Bagenal, T. Dowlind, and W. McKinnon, Cambridge University Press, Cambridge, USA, 639-670, 2004.

Clarke, T. E., C. A. Higgins, J. Skarda, K. Imai, M. Imai, F. Reyes, J. Thieman, T. Jaeger, H. Schmitt, N.P. Dalal, J. Dowell, S. W. Ellingson, B. Hicks, F. Schinzel, and G. B. Taylor, Probing Jovian decametric emission with the Long Wavelength Array station 1, J. Geophys. Res., 119, 9508-9526, 2014.

Clarke, T.E., C.A. Higgins, M. Imai, and K. Imai, Jovian decametric emission with the Long Wavelength Array station 1 (LWA1), in Planetary Radio Emissions VIII, edited by G. Fischer, G. Mann, M. Panchenko, and P. Zarka, Austrian Academy of Sciences Press, Vienna, 31-44, 2017.

Dowell, J., D. Wood, K. Stovall, P. Ray, and G. B. Taylor, The Long Wavelength Array software library, J. Astron. Instrum., 1, id.1250006, 2012.

Dulk, G. A., Io-related radio emission from Jupiter, Science, 148, 1585-1589, 1965.

Dulk, G. A., Y. Leblanc, and A. Lecacheux, The complete polarization state of Io-related radio storms from Jupiter: A statistical study, Astron. Astrophys., 286, 683-700, 1994 .

Flagg, R. S., W. B. Greenman, F. Reyes, and T. D. Carr, A catalog of high resolution Jovian decametric radio noise burst spectra, Volume 1, Dept. of Astronomy, University of Florida, Gainesville, 1991.

Genova, F., and M. G. Aubier, Io-dependent sources of the Jovian decameter emission, Astron. Astrophys., 150, 139-150, 1985.

Hess, S. L. G., P. Zarka, and F. Mottez, Io--Jupiter interaction, millisecond bursts and field-aligned potentials, Planet. Space Sci., 55, 89-99, 2007.

Hess, S. L. G., B. Cecconi, and P. Zarka, Modeling of Io-Jupiter decameter arcs, emission beaming and energy source, Geophys. Res. Lett., 35, L13107, 2008.

Hess, S. L. G., E. Echer, and P. Zarka, Solar wind pressure effects on Jupiter decametric radio emissions independent of Io, Planet. Space Sci., 70, 114-125, 2012.

Imai, K., J. J. Riihimaa, F. Reyes, and T. D. Carr, Measurement of Jupiter's decametric radio source parameters by the modulation lane method, J. Geophys. Res., 107, A6, id.1081, 2002.

Imai, K., C. A. Higgins, M. Imai, and T.E. Clarke, Jupiter's Io-C and Io-B decametric emission source morphology from LWA1 data analysis, in Planetary Radio Emissions VIII, edited by G. Fischer, G. Mann, M. Panchenko, and P. Zarka, Austrian Academy of Sciences Press, Vienna, 89-101, 2017.

Imai, M., A. Lecacheux, T. E. Clarke, C. A. Higgins, M. Panchenko, J. Dowell, K. Imai, A. I. Brazhenko, A. V. Frantsuzenko, and A. A. Konovalenko, The beaming structures of Jupiter's decametric common S-bursts observed from the LWA1, NDA, and URAN2 radio telescopes, Astrophys. J., 826, id.176, 2016. 
Kennedy, D. J., Polarization of the decametric radiation from Jupiter, PhD Thesis, University of Florida, USA, 1969.

Leblanc, Y., On the arc structure of the DAM Jupiter emission, J. Geophys. Res., 86, 8546-8560, 1981.

Leblanc, Y., F. Bagenal, and G. A. Dulk, The Jovian left hand polarized radiation, Astron. Astrophys., 276, 603-613, 1993.

Leblanc, Y., G. A. Dulk, and F. Bagenal, On Io's excitation and the origin of Jupiter's decametric radiation, Astron. Astrophys., 290, 660-673, 1994.

Litvinenko, G., V. Zakharenko, H. O. Rucker, A. A. Konovalenko, V. Vinogradov, V. Nikolaenko, and V. Shaposhnikov, Different types of temporal variations in the decameter emission of Jupiter, European Planetary Science Congress, Rome, Italy, 2010 .

Marques, M. S., P. Zarka, E. Echer, V. B. Ryabov, and M. V. Alves, Statistical analysis of 26 years of observations of decametric radio emissions from Jupiter (abstract), in Planetary Radio Emissions VIII, edited by G. Fischer, G. Mann, M. Panchenko, and P. Zarka, Austrian Academy of Sciences Press, Vienna, p.75, 2017.

Queinnec, J., and P. Zarka, Io-controlled decameter arcs and Io-Jupiter interaction, J. Geophys. Res., 103, A11, 26649-26666, 1998.

Queinnec, J., and P. Zarka, Flux, power, energy and polarization of Jovian S-bursts, Planet. Space Sci., 49, 365-376, 2001.

Riihimaa, J. J., Structured events in the dynamic spectra of Jupiter's decametric radio emission, Astron. J., 73, 265-270, 1968.

Ryabov, V. B., B. P. Ryabov, D. M. Vavriv, P. Zarka, R. Kozhin, V. V. Vinogradov, and V.A. Shevchenko, Jupiter S-bursts: Narrow-band origin of microsecond subpulses, J. Geophys. Res., 112, A09206, 2007.

Warwick, J. W., Dynamic spectra of Jupiter's decametric emission, 1961, Astrophys. J., 137, 41-60, 1963.

Wilkinson, M. H., Io-related Jovian decametric arcs, J. Geophys. Res., 94, 11777-11790, 1989.

Zarka, P., T. Farges, B. P. Ryabov, M. Abada-Simon, and L. Denis, A scenario for Jovian S bursts, Geophys. Res. Lett., 23, 125-128, 1996.

Zarka, P., Auroral radio emissions at the outer planets: Observations and theories, J. Geophys. Res., 103, 20159-20194, 1998. 